\title{
RELAÇÃO ENTRE O POTENCIAL ESPORTIVO DE JOVENS ALU- NOS-ATLETAS E COPING
}

\author{
Diana Izaias Sobreira \\ Universidade Federal de Ouro Preto, Ouro Preto, Minas Gerais, Brasil. \\ Francisco Zacaron Werneck \\ Universidade Federal de Ouro Preto, Ouro Preto, Minas Gerais, Brasil. \\ Emerson Filipino Coelho \\ Universidade Federal de Ouro Preto, Ouro Preto, Minas Gerais, Brasil. \\ Mário Antônio de Moura Simim \\ Universidade Federal do Ceará, Fortaleza, Ceará, Brasil. \\ Eduardo Macedo Penna \\ Universidade Federal do Pará, Castanhal. Pará, Brasil. \\ Luciano Miranda \\ Colégio Militar de Juiz de Fora, Juiz de Fora, Minas Gerais, Brasil. \\ Renato Melo Ferreira \\ Universidade Federal de Ouro Preto, Ouro Preto, Minas Gerais, Brasil.
}

\begin{abstract}
Resumo
Analisar diferenças nas habilidades de coping entre os sexos e o potencial esportivo de alunos-atletas de um colégio militar. Trezentos e oitenta e cinco alunos-atletas responderam o ACSI-28BR. O potencial esportivo foi avaliado pelos treinadores, sendo classificado como baixo e alto. Os meninos apresentaram maiores habilidades de coping do que as meninas. Os meninos de alto potencial esportivo apresentaram maior confiança/motivação, treinabilidade e recurso total de coping, enquanto que as meninas de alto potencial apresentaram maior metas/preparação mental, concentração, confiança/motivação e recurso total de coping. Concluise que as habilidades psicológicas de coping são maiores em alunos-atletas com alto potencial esportivo, variando entre meninos e meninas.
\end{abstract}

Palavras-chave: Coping. Estresse. Escolares. Desempenho. Atletas.

\section{RELATIONSHIP BETWEEN THE SPORTS POTENTIAL OF YOUNG STUDENT- ATHLETES AND COPING}

\begin{abstract}
This study analyzes differences in the coping skills between sexes and the sporting potential of student-athletes of a military college. A total of 385 student-athletes answered the ACSI28BR. The sporting potential was evaluated by coaches, being classified as low and high. Males had higher coping skills than females. Males of high sporting potential showed higher confidence/motivation, trainability and total coping resources; while females with high poten-
\end{abstract}


tial had higher goals/mental preparation, concentration, confidence/motivation and total coping resources. It is concluded that the coping psychological skills are higher in studentathletes with high sporting potential and vary between males and females.

Keywords: Coping. Stress, Scholars, Performance, Athletes.

\title{
RELACIÓN ENTRE EL POTENCIAL DEPORTIVO DE JÓVENES ALUMNOS- ATLETAS Y COPING
}

\begin{abstract}
Resumen
Analizar diferencias en las habilidades de coping entre los sexos y el potencial deportivo de alumnos-atletas de un colegio militar. 385 alumnos-atletas respondieron el ACSI-28BR. El potencial deportivo fue evaluado por los entrenadores, siendo clasificado como bajo y alto. Los niños presentaron mayores habilidades de coping que las niñas. Los niños de alto potencial deportivo presentaron mayor confianza/motivación, capacitación y recurso total de coping; mientras que las niñas de alto potencial presentaron mayores metas / preparación mental, concentración, confianza/motivación y recurso total de coping. Se concluye que las habilidades psicológicas de coping son mayores en alumnos-atletas con alto potencial deportivo, variando entre niños y niñas.
\end{abstract}

Palabras clave: Coping. Estrés. Escolar. Rendimiento. Atletas.

\section{Introdução}

A tarefa de identificar atletas talentosos e promover o seu desenvolvimento tem sido um dos grandes desafios para os pesquisadores e profissionais das Ciências do Esporte (BÖHME, 2007; REES et al., 2016). Dentre os diversos fatores que influenciam o potencial esportivo, há um crescente reconhecimento da importância das características psicológicas como determinantes do desempenho e do desenvolvimento de jovens atletas promissores (ABBOTT; COLLINS, 2004; GEE; MARSHALL; KING, 2010; MACNAMARA; BUTTON; COLLINS, 2010; REES et al., 2016). As evidências atestam a importância das habilidades psicológicas como variáveis discriminantes entre atletas bem-sucedidos daqueles que não alcançaram a elite (VAN YPEREN, 2009; GEE; MARSHALL; KING, 2010).

As características psicológicas de atletas englobam diferentes aspectos que podem influenciar o desempenho esportivo, sendo um dos principais elementos da preparação e seleção do atleta promissor (DE ROSE Jr., 2002). Considera-se seleção de atletas como todo recurso utilizado para apontar indivíduos que apresentam condições de inserção em níveis elevados de exigência de treinamento, objetivando um desempenho ótimo em uma modalidade específica (BÖHME, 2007). Além da aplicação das baterias de testes, o treinador desempenha um papel fundamental na seleção de um jovem atleta promissor, pois sua experiência e conhecimento da modalidade permitem estimar de maneira assertiva o potencial para desempenhos no futuro (SILVA FILHO et al., 2011; VAN YPEREN, 2009).

Com relação aos fatores que podem influenciar o comportamento do atleta no contexto esportivo, estudos têm demonstrado a influência da relação com pais e treinadores (BARROS; DE ROSE Jr., 2006), conflitos internos (DE ROSE Jr.; DESCHAMPS; KORSAKAS, 2001), treinamento (MOREIRA et al., 2010), pressão da torcida (COUTO Jr. et al,, 2007) e nível competitivo (WEIS; ROMANZINI; CARVALHO, 2011; RÉ; DE ROSE Jr.; BOHME, 2004). Logo, para que o atleta lide de forma satisfatória com todas essas variáveis, é preciso que ele desenvolva certas habilidades psicológicas para amenizar a influência negativa de tais variáveis sob o seu desempenho. 
Entre os aspectos psicológicos presentes em qualquer contexto competitivo, a percepção do estresse pode ser considerada um dos fatores cruciais na determinação do desempenho (COIMBRA; MIRANDA; ANDRADE, 2013). Samulski, Noce e Chagas (2009) sugerem que a avaliação das situações estressantes é subjetiva, ou seja, diante da mesma situação, os indivíduos podem reagir de formas distintas ou semelhantes. Essa reação se dá também de acordo com a personalidade, que reflete no modo com que o indivíduo lida com as situações estressoras, com implicação direta sobre o desempenho esportivo (NEVES, 2011).

A literatura denominou como coping o fenômeno de gerenciamento do estresse psicológico (NICHOLLS; POLMAN, 2007). Diante de uma situação estressante, o indivíduo tenta utilizar seus recursos internos de maneira consciente para controlar, reduzir ou resolver as exigências da atividade (COIMBRA, 2011). Para que o atleta tome a decisão correta frente a todas as exigências do jogo e tenha êxito é fundamental que ele saiba lidar com as situações provocadoras de estresse (DE ROSE Jr.; DESCHAMPS; KORSAKAS, 2001). Coimbra et al. (2013) avaliaram jovens atletas de diferentes modalidades esportivas com a finalidade de verificar suas habilidades de coping e concluíram que atletas com maior nível competitivo lidam melhor com adversidades, são mais preparados mentalmente, livres de preocupação, mais confiantes e motivados.

Ao se analisar a relação existente entre estresse e ansiedade em jovens, percebe-se que a literatura diverge em relação a diferenças entre os sexos. Alguns estudos (CRAFT et al., 2003; GONÇALVES; BELO, 2007) apontam níveis mais elevados de ansiedade e estresse nas meninas, enquanto outros estudos (CAPUTO; ROMBALDI; SILVA, 2017) identificaram níveis semelhantes entre meninos e meninas quando considerado o início da carreira esportiva destes atletas. Ao considerar exclusivamente o constructo de coping, Coimbra et al. (2013) verificaram que meninos apresentam melhor enfrentamento do estresse particularmente nas dimensões desempenho sob pressão, lidar com adversidades, metas/preparação mental e confiança/motivação. Diferenças nas habilidades psicológicas de coping também foram reportadas por Nicholls e Polman (2007), após revisão sistemática da literatura. No entanto, informações sobre esta temática em jovens alunos-atletas oriundos de colégios militares são escassas.

A capacidade de atuar sob pressão é necessária para atletas atingirem suas metas e suportarem as exigências esportivas (KENT et al., 2018). No caso de jovens atletas, fatores complexos e multifatoriais contribuem para que experimentem diferentes estressores, tais como relacionamento com colegas de equipe, tarefas escolares, mudanças físicas e emocionais (GARCIA, 2010). Além disso, investigar como jovens atletas lidam ou respondem a esses estressores contribui para compreensão do coping (NICHOLLS; POLMAN, 2007) e, com isso, podem melhorar seu desempenho mediante situações estressoras (KENT et al., 2018; NICHOLLS; POLMAN, 2007). Adicionalmente, destaca-se que atletas de sucesso são aqueles que possuem estratégias de enfrentamento (coping) mais eficientes perante situações de estresse inerentes à prática esportiva (ROSSI et al., 2016).

Existe evidência científica de que habilidades psicológicas, tais como motivação, confiança, resiliência e coping são importantes para o desenvolvimento de superatletas (REES et al., 2016). Entretanto, não se sabe até que ponto as habilidades de coping estariam associadas ao potencial esportivo de jovens promissores. Diante dessas considerações, o objetivo do presente estudo foi analisar diferenças nas habilidades psicológicas de coping em alunos-atletas de um colégio militar, em função do sexo e do seu potencial esportivo avaliado pelo treinador.

\section{Metodologia}

Trata-se de uma pesquisa quantitativa, descritiva, de corte transversal. A população alvo foi os escolares do Sistema Colégio Militar do Brasil. Por conveniência, o estudo foi reali- 
zado no Colégio Militar de Juiz de Fora (CMJF). Participaram do estudo 385 alunos-atletas, com idade entre 11 e 18 anos (14,7 $\pm 2,0$ anos). Foi estabelecido como critério de inclusão que os mesmos deveriam participar das aulas de Educação Física e dos treinamentos das modalidades esportivas, individuais e/ou coletivas, regularmente. Foram excluídos os escolares que não entregaram o termo de consentimento assinado pelo responsável ou que se recusaram a participar e aqueles que não preencheram adequadamente o questionário. Os alunos foram dispostos em uma sala, onde receberam instruções do pesquisador quanto ao preenchimento do questionário. Este estudo é parte integrante do "Projeto Atletas de Ouro: Avaliação Multidimensional e Longitudinal do Potencial Esportivo de Jovens Atletas" (WERNECK et al., 2017), aprovado pelo Comitê de Ética em Pesquisa da Universidade Federal de Ouro Preto (CAAE: 32959814.4.1001.5150).

Para a avaliação do coping, foi utilizado o Athletic Coping Skills Inventory - 28 na sua versão em português (ACSI-28BR) (COIMBRA, 2011). Este instrumento é composto por 28 questões, divididas em sete subescalas, cada uma composta por quatro itens. As respostas se dão em escala de 0 a $3(0=$ quase nunca, $1=$ às vezes, $2=$ frequentemente, $3=$ quase sempre $)$, com escore final, variando de 0 a 12 pontos. A soma das subescalas é chamada recurso pessoal de coping. As subescalas do ACSI-28BR (COIMBRA, 2011) estão descritas no Quadro 1, abaixo.

Quadro 1 - Resumo descritivo das subescalas do ACSI-28BR e seus respectivos itens

\begin{tabular}{lll}
\hline \multicolumn{1}{c}{ Subescala } & \multicolumn{1}{c}{ Descrição } & \multicolumn{1}{c}{ Itens } \\
\hline Lidar com adversidades & Rápida recuperação frente ao fracasso (controle) & $5,17,21,24$ \\
\hline Desempenho sob pressão & Elevado desempenho frente ao desafio & $6,18,22,28$ \\
\hline Metas/Preparação Mental & Metas específicas e se preparo mental (prática) & $1,8,13,20$ \\
\hline Concentração & Pouca distração em qualquer situação & $4,11,14,25$ \\
\hline Livre de Preocupação & Foco, falta de preocupação frente ao supérfluo & $7^{*}, 12^{*}, 19^{*}$, \\
& & $23^{*}$ \\
\hline Confiança/Motivação & Confiança e motivação no desempenho & $2,9,16,26$ \\
\hline Treinabilidade & Aprendizado alto, aceita crítica (crescimento) & $3^{*}, 10^{*}, 15$, \\
& & 27 \\
\hline
\end{tabular}

*Itens em itálico devem ser invertidos durante a soma dos escores para o cálculo da subescala.

Fonte: Coimbra (2011).

Para a avaliação do potencial esportivo, participaram 10 professores-treinadores do colégio das seguintes modalidades esportivas: futebol, voleibol, corrida de orientação, natação, esgrima, triatlo militar, basquetebol, handebol e atletismo, com idade entre 31 a 53 anos (41 \pm 8 anos) e tempo de experiência entre dois a 30 anos (12,5 $\pm 9,8$ anos). A formação acadêmica dos mesmos era graduação (2), especialização (3) e mestrado (5), sendo a maioria ex-atletas de nível estadual e nacional. Foi solicitado aos professores-treinadores que avaliassem o potencial esportivo dos alunos-atletas, de acordo com a expectativa futura depositada em cada um deles. Dessa maneira, o potencial esportivo foi classificado em: baixo potencial - aqueles indivíduos classificados pelo treinador como potencial esportivo muito fraco (1), fraco (2) e razoável (3); alto potencial - aqueles indivíduos classificados pelo treinador como potencial esportivo bom (4) e muito bom (5);

Estatística descritiva (média \pm desvio padrão) foi utilizada para sumarizar os resultados das variáveis do estudo. O Teste " $t$ " de Student para amostras independentes foi empregado para comparar as habilidades de coping entre os sexos e potencial esportivo (baixo vs. alto potencial). O nível de significância adotado foi de $5 \%(\mathrm{p}<0,05)$ e utilizado Software SPSS versão 20. 


\section{Resultados}

A Tabela 1 apresenta o resultado das seis dimensões do instrumento para a amostra como um todo e a comparação entre os sexos. Foram observados maiores valores para os meninos em relação às meninas nas subescalas lidar com adversidades, desempenho sob pressão, concentração, livre de preocupação e confiança e motivação, resultando também em maior recurso pessoal de coping.

Tabela 1 - Resultado das subescalas de coping de alunos-atletas

\begin{tabular}{ccccc}
\hline & Geral & Masculino & Feminino & p-valor \\
& $(\mathrm{n}=385)$ & $(\mathrm{n}=218)$ & $(\mathrm{n}=167)$ & \\
\hline Lidar com Adversidades & $1,54 \pm 0,67$ & $1,62 \pm 0,60$ & $1,43 \pm 0,74$ & $0,006^{*}$ \\
Desempenho sob Pressão & $1,11 \pm 0,76$ & $1,24 \pm 0,75$ & $0,94 \pm 0,74$ & $<0,001^{*}$ \\
Metas/Preparação Mental & $1,48 \pm 0,71$ & $1,48 \pm 0,73$ & $1,48 \pm 0,69$ & 0,98 \\
Concentração & $1,69 \pm 0,61$ & $1,77 \pm 0,57$ & $1,58 \pm 0,64$ & $0,002^{*}$ \\
Livre de Preocupação & $1,38 \pm 0,73$ & $1,47 \pm 0,71$ & $1,27 \pm 0,75$ & $0,007^{*}$ \\
Confiança/Motivação & $1,80 \pm 0,63$ & $1,86 \pm 0,62$ & $1,72 \pm 0,64$ & $0,03^{*}$ \\
Treinabilidade & $2,30 \pm 0,57$ & $2,32 \pm 0,53$ & $2,27 \pm 0,60$ & 0,41 \\
Recurso Pessoal de Coping & $11,32 \pm 2,82$ & $11,79 \pm 2,71$ & $10,73 \pm 2,85$ & $<0,001^{*}$ \\
\hline
\end{tabular}

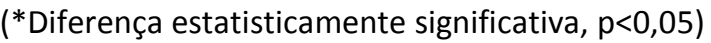

Fonte: Elaborada pelos autores (2018).

Os resultados da comparação do potencial esportivo (alto e baixo potencial) estão apresentados na Tabela 2, para a amostra como um todo e separadamente por sexo. Destaca-se que para os meninos, as subescalas confiança / motivação, treinabilidade e recurso pessoal de coping apresentaram diferença significativa. Enquanto que para as meninas foram as dimensões metas / preparação mental, concentração, confiança / motivação e recurso pessoal de coping. 
Tabela 2 - Média \pm desvio-padrão das habilidades psicológicas de coping de alunosatletas, classificados pelos treinadores quanto ao potencial esportivo com a participação nos treinamentos

\begin{tabular}{|c|c|c|c|}
\hline Geral & $\begin{array}{l}\text { Baixo Potencial } \\
(\mathrm{n}=198)\end{array}$ & $\begin{array}{l}\text { Alto Potencial } \\
\qquad(\mathrm{n}=187)\end{array}$ & p-valor \\
\hline Lidar com Adversidades & $1,49 \pm 0,72$ & $1,58 \pm 0,63$ & 0,24 \\
\hline Desempenho sob Pressão & $1,05 \pm 0,78$ & $1,17 \pm 0,75$ & 0,11 \\
\hline Metas/Preparação Mental & $1,42 \pm 0,72$ & $1,55 \pm 0,71$ & 0,08 \\
\hline Concentração & $1,62 \pm 0,67$ & $1,77 \pm 0,54$ & $0,01 *$ \\
\hline Livre de Preocupação & $1,32 \pm 0,74$ & $1,44 \pm 0,74$ & 0,11 \\
\hline Confiança/Motivação & $1,67 \pm 0,66$ & $1,93 \pm 0,59$ & $0,000 *$ \\
\hline Treinabilidade & $2,24 \pm 0,59$ & $2,38 \pm 0,53$ & $0,01 *$ \\
\hline Recurso Pessoal de Coping & $10,85 \pm 2,96$ & $11,84 \pm 2,64$ & $0,001 *$ \\
\hline Masculino & $\begin{array}{c}\text { Baixo Potencial } \\
(\mathrm{n}=97)\end{array}$ & $\begin{array}{l}\text { Alto Potencial } \\
\qquad(\mathrm{n}=121)\end{array}$ & p-valor \\
\hline Lidar com Adversidades & $1,60 \pm 0,66$ & $1,63 \pm 0,56$ & 0,76 \\
\hline Desempenho sob Pressão & $1,16 \pm 0,79$ & $1,30 \pm 0,74$ & 0,18 \\
\hline Metas/Preparação Mental & $1,48 \pm 0,76$ & $1,48 \pm 0,71$ & 0,99 \\
\hline Concentração & $1,74 \pm 0,60$ & $1,81 \pm 0,54$ & 0,37 \\
\hline Livre de Preocupação & $1,40 \pm 0,70$ & $1,53 \pm 0,72$ & 0,18 \\
\hline Confiança/Motivação & $1,73 \pm 0,65$ & $1,97 \pm 0,58$ & $0,005^{*}$ \\
\hline Treinabilidade & $2,24 \pm 0,55$ & $2,39 \pm 0,52$ & $0,04 *$ \\
\hline Recurso Pessoal de Coping & $11,38 \pm 2,81$ & $12,14 \pm 2,64$ & $0,04 *$ \\
\hline Feminino & $\begin{array}{l}\text { Baixo Potencial } \\
\qquad(\mathrm{n}=101)\end{array}$ & $\begin{array}{l}\text { Alto Potencial } \\
\qquad(\mathrm{n}=66)\end{array}$ & p-valor \\
\hline Lidar com Adversidades & $1,39 \pm 0,75$ & $1,48 \pm 0,74$ & 0,46 \\
\hline
\end{tabular}




\begin{tabular}{cccc} 
Desempenho sob Pressão & $0,94 \pm 0,76$ & $0,94 \pm 0,73$ & 0,97 \\
Metas/Preparação Mental & $1,36 \pm 0,68$ & $1,66 \pm 0,70$ & $0,007^{*}$ \\
Concentração & $1,50 \pm 0,71$ & $1,70 \pm 0,54$ & $0,05^{*}$ \\
Livre de Preocupação & $1,25 \pm 0,77$ & $1,29 \pm 0,74$ & 0,75 \\
Confiança/Motivação & $1,62 \pm 0,66$ & $1,87 \pm 0,61$ & $0,02^{*}$ \\
Treinabilidade & $2,24 \pm 0,64$ & $2,35 \pm 0,54$ & 0,28 \\
Recurso Pessoal de Coping & $10,34 \pm 3,02$ & $11,30 \pm 2,59$ & $0,03^{*}$ \\
\hline
\end{tabular}

(*Diferença estatisticamente significativa, $\mathrm{p}<0,05$ )

Fonte: Elaborada pelos autores (2018).

\section{Discussão}

O objetivo do estudo foi analisar as diferenças nas habilidades psicológicas de coping entre alunos-atletas em função do sexo e do seu potencial esportivo (alto e baixo potencial), avaliado pelo treinador. O principal resultado aponta que as dimensões confiança/motivação e recurso pessoal de coping foram as que apresentaram diferenças em ambos os sexos, além disso, a dimensão treinabilidade apresentou diferença para os meninos e as metas/preparação mental e concentração apresentaram diferenças para as meninas.

Na comparação das habilidades de coping entre os sexos (Tabela 1), cinco das sete subescalas (lidar com adversidades, desempenho sob pressão, concentração, livre de preocupação, confiança/motivação), além do recurso pessoal de coping apresentaram diferenças significativas, com média maior para os meninos. O estudo de Coimbra et al. (2013) corrobora em parte os resultados do presente estudo, já que identificou escores maiores para os meninos em relação as meninas em quatro dimensões (desempenho sob pressão, lidar com adversidades, metas/preparação mental, confiança/ motivação). Vieira et al. (2013) identificaram níveis maiores de coping nos meninos em todas dimensões do coping. Tal resultado pode ser justificado devido as meninas apresentarem maiores níveis de ansiedade pré-competitiva (DE ROSE Jr.; VASCONCELOS, 1997; CRAFT et al., 2003; GONÇALVES; BELO, 2007). O aparecimento da ansiedade antes das competições acarreta em aumento da preocupação do indivíduo quanto a resultados desfavoráveis (HERNANDEZ; GOMES, 2002), influenciando, diretamente, nas estratégias de enfrentamento.

O contexto competitivo apresenta um padrão de desempenho no qual o indivíduo é comparado / avaliado por pessoas que conhecem as características necessárias para a melhoria do rendimento (DE ROSE Jr.; VASCONCELOS, 1997; FALK et al., 2004). Dessa forma, a partir da classificação do treinador quanto ao potencial esportivo (Tabela 2), ao analisar a amostra de forma geral, percebe-se que as dimensões concentração, confiança/motivação, treinabilidade, além do recurso pessoal de coping foram as que apresentaram diferenças significativas em analisar baixo vs. alto potencial esportivo. Além disso, verificou-se que os meninos classificados como alto potencial se apresentaram mais confiantes / motivados, com níveis maiores de treinabilidade e lidam melhor com o estresse percebido no ambiente competitivo, de modo que seu desempenho venha sofrer pouca interferência negativa. As meninas classifi- 
cadas como alto potencial também lidam melhor com o estresse percebido e são mais confiantes/motivadas, além disso, obtiveram maiores níveis de concentração e metas /preparação mental. Essas dimensões avaliam, respectivamente, o foco e a atenção direcionados a um único objeto e o plano de competição idealizado pelo atleta antes mesmo deste começar, o que corrobora em parte com Rossi et al. (2016), que encontraram diferença significativa em três dimensões (desempenho sob pressão, confiança/motivação e concentração) entre atletas de futebol feminino de alto rendimento e amadoras. A partir desses resultados, percebemos que, para se atingir um possível alto rendimento, é de grande importância ter os níveis de confiança e motivação elevados. De acordo com Zanetti, Lavoura e Machado (2008), quanto maior a percepção do indivíduo quanto às suas capacidades diante dos resultados positivos, mais confiantes e motivados eles se tornam.

As sessões de treino podem ser um dos fatores que aumentam os níveis de estresse, porém é um episódio importante, no qual atleta e treinador buscam se adaptarem às situações inerentes da competição. Além disso, durante o treinamento a longo prazo, ajustam as estratégias de enfrentamento de modo com que, ao chegarem na competição, possam manter seu nível de estresse baixo e/ou lide com ele de forma que seu desempenho não seja prejudicado (BELEM et al., 2016; BIM et al., 2014; TINELI et al., 2011).

Esses resultados refletem a ideia de que os meninos são mais competitivos e possuem maior motivação intrínseca (MIRANDA et al., 2015), já as meninas, apesar de gostarem de treinar, precisam de motivação extrínseca. A falta dessa motivação pode ser um fator determinante para o abandono esportivo, como observado no estudo de Bara Filho e Garcia (2008), em que as meninas são as que mais evadem da prática esportiva. Pires et al. (2016) afirmam que o estresse gerado pelos treinamentos e competições, quando não gerenciados de forma adequada, podem prejudicar o desempenho dos atletas e até mesmo provocar o abandono da modalidade. Ademais, pode-se associar este resultado ao conceito de esporte participativo, onde o maior propósito não é vencer ou ser o melhor, mas sim se divertir e interagir ( $\mathrm{BOH}-$ ME, 2000). Com base nisso, o treinador tem uma boa ferramenta para ajustar os treinamentos buscando o melhor de cada atleta, diminuindo o efeito negativo do estresse, e também melhorando as habilidades de coping dos indivíduos que apresentam médias inferiores, pois esse pode ser o fator principal do seu baixo rendimento esportivo.

A partir dessas informações, tem-se a necessidade de apontar algumas limitações deste estudo, pois além de conhecer as estratégias de enfrentamento do estresse, é necessário distinguir os fatores causadores do próprio estresse com a finalidade de identificar quais as variáveis de coping são mais apropriadas em determinadas situações. Além disso, é preciso realizar uma análise separadamente pelo tipo de modalidade (individual e coletiva), pois estas apresentam diferentes características e situações estressoras. Sugere-se, também, que os dados sejam coletados na fase competitiva onde, pressupõe-se, os níveis de estresse estarão maiores. O tempo de experiência esportiva também é uma possível variável interveniente nas habilidades de coping e não foi controlada no presente estudo.

É importante destacar que na fase inicial de identificação de potenciais talentos esportivos torna-se importante conhecer os motivos que levam a criança a praticar o esporte, o seu nível de autoconfiança, autoestima e senso de competência, bem como o seu potencial para lidar com o estresse e as demandas do esporte. Alguns autores atestam que o principal indicador da capacidade de desenvolvimento no esporte parece ser de natureza psicocomportamental e, portanto, determinados perfis psicológicos estariam associados a maiores desempenhos (ABBOT; COLLINS, 2004). Porém, ressalta-se que as habilidades de coping não devem ser utilizadas com a finalidade de seleção de atletas (MIRANDA et al., 2018).

Neste sentido, a utilização de instrumentos para avaliação das características psicológicas de jovens atletas deve ter uma função educativa/formativa, ligada diretamente ao desen- 
volvimento dos jovens atletas, uma vez que permitem a identificação de forças e fraquezas no perfil do atleta que podem ser trabalhadas pelos técnicos através de intervenções específicas. O mapeamento das características psicológicas de jovens atletas permitirá o direcionamento de apoio e recursos àqueles indivíduos que têm maior potencial de atingir o sucesso no esporte bem como dar a oportunidade para o desenvolvimento das potencialidades de crianças e jovens que apresentem necessidades de desenvolvimento em seu perfil, por meio de um processo sistemático e contínuo, com foco na inclusão, envolvendo pais, técnicos e demais profissionais do esporte. Estudos longitudinais são necessários para se avaliar como evoluem as habilidades de coping ao longo do tempo, bem como os efeitos do treinamento.

\section{Conclusão}

O presente estudo verificou que as habilidades psicológicas de coping são maiores nos alunos-atletas com alto potencial esportivo, variando entre meninos e meninas. Os meninos classificados como alto potencial esportivo, quando comparados aos de baixo potencial, lidam melhor com o estresse, são mais confiantes e motivados e apresentam maior treinabilidade. As meninas de alto potencial esportivo também lidam melhor com o estresse, são mais confiantes e motivadas, além de serem mais concentradas e terem apresentado níveis maiores de metas e preparação mental.

\section{Referências}

ABBOT, A.; COLLINS, D. Eliminating the dichotomy between theory and practice in talent identification and development: considering the role of psychology. Journal of Sports Sciences, London, v.22, n.5, p.395-408, 2004.

BARA FILHO, M. G.; GARCIA, F. G. A competição como fonte de estresse no esporte. Revista Brasileira de Educação Física e Esportes, São Paulo, v.22, n.4, p.293-300, out./dez. 2008.

BARROS, J. C. T. S.; DE ROSE Jr., D. Situações de stress na natação infanto-juvenil: atitudes de técnicos e pais, ambientes competitivos e momentos que antecedem a competição. Revista Brasileira de Ciência e Movimento, São Paulo, v. 14, n. 4, p. 79-86, jun. 2006.

BELEM, I.; COSTA, L. C. A.; BOTH, J.; PASSOS, P. C. B.; VIEIRA, J. L. L. O estresse no MMA: As estratégias de enfrentamento podem melhorar o desempenho dos lutadores? Revista Brasileira de Medicina do Esporte, São Paulo, v. 22, n. 4, p. 287-290, ago. 2016.

BIM, R.; DO NASCIMENTO Jr, J. R. A.; AMORIM, A. C.; VIEIRA, J. L. L.; VIEIRA, L. F. Estratégias de coping e sintomas de burnout em atletas de futsal de alto rendimento. Revista Brasileira Ciência e Movimento, São Paulo, v. 22, n. 3, p. 69-75, 2014.

BOHME, M. T. S. O treinamento a longo prazo e o processo de detecção, seleção e promoção de talentos esportivos. Revista Brasileira de Ciências do Esporte, Campinas, v. 21, n. 2/3, p. 4-10, jan./maio 2000.

BOHME, M. T. S. O tema talento esportivo na ciência do esporte. Revista Brasileira de Ciência e Movimento, São Paulo, v. 1, n. 15, p. 119-126, jul. 2007. 
CAPUTO, E. L.; ROMBALDI, A. J.; SILVA, M. C. Sintomas de estresse pré-competitivo em atletas adolescentes de handebol. Revista Brasileira de Ciência do Esporte, Campinas, v. 39, n. 1, p. 68-72, 2017.

COIMBRA, D. R. Processo de validação do questionário "Athletic Coping Skills Invetory-28" para a língua portuguesa do Brasil. 2011. 144 f. Dissertação (Mestrado em Educação Física), Universidade Federal de Juiz de Fora, Juiz de Fora, 2011. Disponível em: https://repositorio.ufjf.br/jspui/handle/ufjf/2171. Acesso em: 30 ago. 2016.

COIMBRA, D. R.; MIRANDA, R.; ANDRADE, A. Habilidades psicológicas de coping em atletas brasileiros. Motricidade, [S.1.], v. 9, n. 1, p. 95-106, dez. 2013.

COUTO Jr, J. M.; MORENO, R. M.; DE SOUZA, A. F.; PRADO, M. M.; MACHADO, A. A. A influência da torcida na performance de jogadores brasileiros de futsal: Um viés da Psicologia do Esporte. Motriz, São Paulo, v. 13, n. 4, p. 359-265, dez. 2007.

CRAFT, L. L.; MAGYAR, T. M.; BECKER, B. J.; FELTS, C. L. The relationship between the competitive state anxiety inventory 2- and sport performance: a meta-analysis. Journal of Sport \& Exercise Psychology, London, v. 25, n. 1, p. 44-65, 2003.

DE ROSE Jr., D.; DESCHAMPS, S. R.; KORSAKAS, P. O jogo como fonte de stress no basquetebol infanto-juvenil. Revista Portuguesa de Ciências do Desporto, Porto, v. 1, n. 2, p.36-44, jan. 2001.

DE ROSE Jr, D. A competição como fonte de estresse no esporte. Revista Brasileira Ciência e Movimento, São Paulo, v. 10, n. 4, p. 19-26, out. 2002.

DE ROSE Jr, D.; VASCONCELOS, E. G. Ansiedade traço competitiva e atletismo: um estudo com atletas infanto-juvenis. Revista Paul. Educação Física, São Paulo, v. 11, n. 2, p. 148154, jul./dez. 1997.

FALK, B.; LIDOR, R.; LANDER, Y.; LANG, B. Talent identification and early development of elite water-polo players: a 2-year follow-up study. Journal of Sports Sciences, London, v. 22, n. 1, p. 347-355, 2004.

GARCIA, C. Conceptualization and measurement of coping during adolescence: A review of the literature. Journal of Nursing Scholarship, Indianapolis, v. 42, n. 2, p. 166-85, Jun. 2010. doi: 10.1111/j.1547-5069.2009.01327.x

GEE, C.J.; MARSHALL, J.C.; KING, J.F. Should coaches use personality assessments in the talent identification process? A 15 year predictive study on professional hockey players. International Journal of Coaching Science, Seoul, v.4, n.1, p.25-34, 2010.

GONÇALVES, M. P.; BELO, R. P. Ansiedade-traço competitiva: diferenças quanto ao gênero, faixa etária, experiências em competições e modalidade esportiva em jovens atletas. PsicoUSF, Campinas, v. 12, n. 2, p. 301-307, jul./dez. 2007. 
HERNANDEZ, J. A. E.; GOMES, M. M. Coesão grupal, ansiedade pré-competitiva e o resultado dos jogos em equipes de futsal. Revista Brasileira Ciência do Esporte, Campinas, v. 24, n. 1, p. 139-150, set. 2002.

KENT, S.; DEVONPORT, T. J.; LANE, A. M.; NICHOLLS, W.; FRIESEN, A. P. The effects of coping interventions on ability to perform under pressure. Journal of Sports Science Medicine, Bursa, v. 17, n. 1, p. 40-55, mar. 2018.

MACNAMARA, A.; BUTTON, A., COLLINS, D. The role of psychological characteristics in facilitating the pathway to elite performance. Part 1: Identifying mental skills and behaviours. The Sport Psychologist, Champaign, v.24, n. 1, p. 52-73, 2010.

MIRANDA, R.; COIMBRA, C. R.; MIRANDA Jr, M. U.; RUSSO, A. F.; ANDRADE, A.; BARA FILHO, M. Habilidades de coping em atletas escolares brasileiros. Caderno de Educação Física e Esporte, Paraná, v. 13, n. 1, p. 31-38, jun. 2015.

MIRANDA, R.; COIMBRA, D. R.; BARA FILHO, M. G.; MIRANDA JUNIOR, M. V.; ANDRADE, A. Brazilian version (ACSI-28BR) of atletic coping skills inventory-28. Revista Brasileira de Medicina no Esporte, São Paulo, v. 24, n.2, p.130-134, maio/ abr., 2018.

MOREIRA, A.; FREITAS, C. G.; NAKAMURA, F. Y.; AOKI, M. S. Percepção de esforço da sessão e a tolerância ao estresse em jovens atletas de voleibol e basquetebol. Revista Brasileira de Cineantropometria e Desempenho Humano, Florianópolis, v. 12, n. 5, p. 345351, jan. 2010.

NEVES, F. S. Avaliação da dinâmica resiliente em atletas paraibanos. 2011. 165 f. Dissertação (Mestrado em Psicologia Social) - Universidade Federal da Paraíba, João Pessoa, 2011. Disponível em: http://tede.biblioteca.ufpb.br:8080/handle/tede/7026. Acesso em: 04 out. 2016.

NICHOLLS, A. R.; POLMAN, R. C. Coping in sport: a systematic review. Journal of Sports Science, London, v. 25, n. 1, p. 11-31, 2007.

PIRES, D. A.; BARA FILHO, M. G.; DEBIEN, P. B.; COIMBRA, D. R., UGRINOWTSCH, H. Burnout e coping em atletas de voleibol: uma análise longitudinal. Revista Brasileira de Medicina do Esporte, São Paulo, v. 22, n. 4, p.277-281, jul./ago. 2016.

RÉ, A. H. N.; DE ROSE Jr, D.; BOHME, M. T. S. Stress e nível competitivo: Considerações sobre jovens praticantes de futsal. Revista Brasileira de Ciência e Movimento, Brasília, v. 12, n. 4, p. 83-87, dez. 2004.

REES, T.; HARDY, L.; GÜLLICH, A.; ABERNETHY, B.; CÔTÉ, J.; WOODMAN, T.; ... WARR, C. The great British medalists project: A review of current knowledge on the development of the world's best sporting talent. Sports Medicine, Auckland, v.46, n.8, p.1041$1058,2016$.

ROSSI, M. R.; VITORINO, L. M.; SALLES, R. P., CORTEZ, P. J. O. Estratégias de coping em atletas de futebol feminino: um estudo comparativo. Revista Brasileira de Medicina do Esporte, São Paulo, v. 22, n. 4, p. 282-286, ago. 2016. 
SAMULSKI, D.; NOCE, F.; CHAGAS, M. H. Estresse. In: SAMULSKI, D. M. Psicologia do Esporte: conceitos e novas perspectivas. Barueri: Manole, 2009, p. 231-264.

SILVA FILHO, F. J.; LUGUETTI, C. N.; PAES, F. O. P.; BOHME, M. T. S. Critérios para detecção e seleção de jovens atletas de basquetebol na cidade de São Paulo. Revista Mackenzie de Educação Física e Esporte, São Paulo, v. 10, n. 2, p. 64-73, 2011.

TINELI, D.; ROVER, C.; FIN, G.; NODARI Jr, R. J.; FIEDLER, M. M.; BARETTA, E. Ansiedade-traço pré-competitiva: um estudo com atletas de judô. Unoesc \& Ciência - ACBS, Joaçaba, v. 2, n. 2, p. 107-116, jul./dez. 2011

VAN YPEREN, N. W. Why some make it and others do not: Identifying psychological factors that predict career success in professional adult soccer. The Sport Psychologist, Champaign, v.23, p. 317-329, 2009.

VIEIRA, L. F.; CARUZZO, N. M.; AIZAVA, P. V.; RIGONI, P. A. Análise da síndrome de "burnout" e das estratégias de "coping" em atletas brasileiros de vôlei de praia. Revista Brasileira de Educação Física e Esporte, São Paulo, v. 27, n. 2, p. 269-276, 2013.

WEIS, G. F.; ROMANZINI, C. L. P.; CARVALHO, V. Competições esportivas como fonte de estresse: análise das equipes infanto-juvenil do projeto Cestinha em Santa Cruz do Sol/RS. Revista Brasileira de Ciência e Movimento, São Paulo, v. 19, n. 1, p. 58-65, out. 2011.

WERNECK, F.Z.; FERREIRA, R.M.; COELHO, E.F.; SOBREIRA, D.I; DE PAULA, H.L.; MIRANDA, L.; RIBAS, P. R.; FIGUEIREDO, A. J. Projeto atletas de ouro: validade e estabilidade do diagnóstico do potencial esportivo em escolares de um colégio militar. In: IV Fórum Científico da ESEFEx. Revista de Educação Física, Rio de Janeiro, v.86, n.2, p. 139-141, 2017.

ZANETTI, M. C.; LAVOURA, T. N.; MACHADO, A. A. Motivação no esporte infanto juvenil. Conexões: Revista da faculdade de Educação Física da UNICAMP, Campinas, v.8, n. especial, p. 438-447, jul. 2008.

Apoio Financeiro da Universidade Federal de Ouro Preto, edital 006/2016-PIP-2S/UFOP. Apoio logístico do Colégio Militar de Juiz de Fora - Acordo de Cooperação EME 18-055-00. Apoio financeiro por meio de emenda parlamentar no 36820009, PLN 0020/207-LOA.

Recebido em: 10/06/2018

Revisado em: 14/09/2018

Aprovado em: 18/10/2018

Endereço para correspondência:

renato.mf@hotmail.com

Renato Melo Ferreira

Universidade Federal de Ouro Preto,

Centro Desportivo, Morro do Cruzeiro

Bauxita

35400000 - Ouro Preto, MG - Brasil. 